\title{
THE ARCTIC Differences and similarities between users of traditional healing and UNIVERSITY OF N ORWAY other CAM modalities in the seventh survey of the Troms $\varnothing$ Study
}

\author{
Agnete E. Kristoffersen ${ }^{1}$, Ann Ragnhild Broderstad ${ }^{2}$, Frauke Musial ${ }^{1}$ and Trine Stub ${ }^{1}$ \\ 1 The National research center in complementary and alternative medicine (NAFKAM), Department of Community Medicine, UiT The Arctic University of Norway \\ 2 Centre for Sami Health Research, Department of Community Medicine, UiT The Arctic University of Norway
}

\section{Background}

Both traditional healing (TH) and other Complementary and Alternative Medicine (CAM) modalities are widely used in northern Norway. While TH has been part of lay health care system for centuries, other CAM modalities like acupuncture, homeopathy, reflexology etc. were introduced more recently. The aim of this study was to explore the differences and the similarities regarding self-reported health, gender, age, education, income, religiosity and ethnicity between users of TH and users of other CAM modalities in a northern Norwegian population.

\section{Methods}

The data were drawn from the seventh survey of the Troms $\varnothing$ Study, a longitudinal population-based cohort study conducted in 2015-2016. All inhabitants of Troms $\varnothing$ aged 40 and above were invited $(n=32,591)$ and $n=21,083$ accepted the invitation (response rate 65\%). Data were collected through three self-administered questionnaires and a comprehensive clinical examination. Pearson chi-square tests and one-way ANOVA tests were used to describe differences between the groups while binary logistic regressions were used for adjusted values.
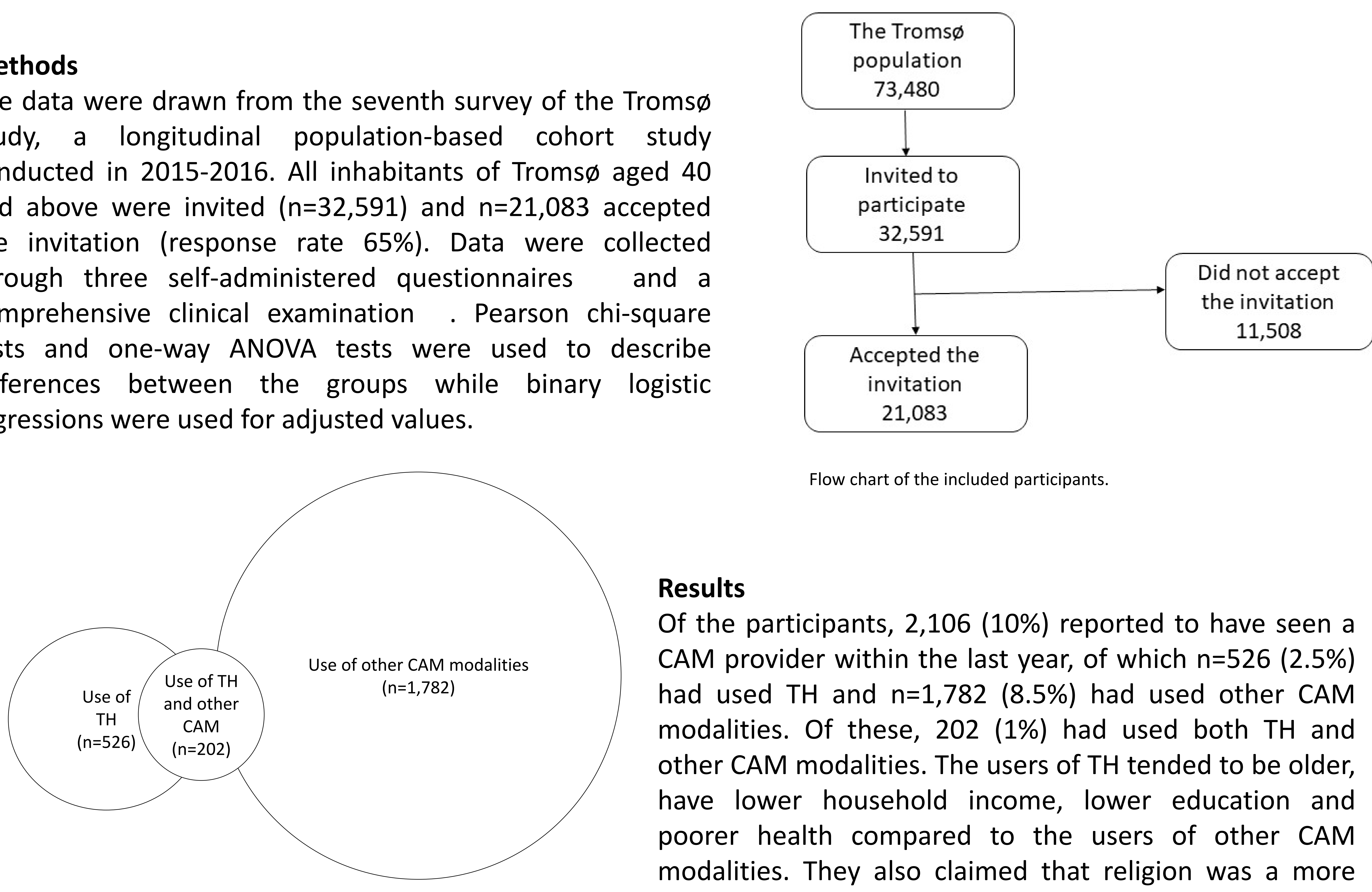

Flow chart of the included participants.

\section{Conclusion}

The different associations for use of TH and other CAM modalities as well as the low proportion of participants reporting to have used both $\mathrm{TH}$ and other CAM modalities, indicates that TH and other CAM modalities are used by different group of people.

\section{Results}

Of the participants, $2,106(10 \%)$ reported to have seen a CAM provider within the last year, of which $n=526(2.5 \%)$ had used $\mathrm{TH}$ and $\mathrm{n}=1,782(8.5 \%)$ had used other CAM modalities. Of these, 202 (1\%) had used both TH and other CAM modalities. The users of TH tended to be older, have lower household income, lower education and poorer health compared to the users of other CAM modalities. They also claimed that religion was a more important part of their life. We found no significant differences between users of $\mathrm{TH}$ and other CAM modalities regarding gender, although men was slightly more likely to use TH compared to other CAM modalities $(p=0.085)$. Women used, however both TH and other CAM modalities more frequently than men did. Participants living without a spouse/partner were most likely to have used a combination of TH and other CAM modalities. 